\title{
Comment on 'Abortion care services delivered from a community sexual and reproductive health setting: views of health care professionals': authors' response
}

We thank Dr Kell and Ms McMahon for their comments ${ }^{1}$ on our article ${ }^{2}$ and for sharing their experience of running an abortion service from a community setting. It is reassuring to learn that both staff and patients welcomed this service and that both contraceptive provision and sexually transmitted infection diagnosis have improved as a result. We hope other clinicians providing abortion care services may be encouraged to consider the potential benefits of providing such services from a community setting.

Lucy Michie, MRCOG, DFSRH

Specialty Trainee in Sexual and Reproductive Health, Sandyford Initiative, Glasgow, UK;

michieluc@yahoo.co.uk

Sharon Cameron, MRCOG, MFSRH

Consultant Gynaecologist, Chalmers Sexual and Reproductive Health Centre, Edinburgh, UK; Sharon.Cameron@ed.ac.uk

Anna Glasier, FRCOG, FFSRH

Honorary Professor, Department of Reproductive and Developmental Sciences, University of Edinburgh, Edinburgh, UK; Anna.Glasier@ed.ac.uk

Competing interests None.

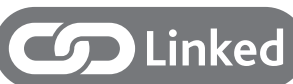

- http://dx.doi.org/10.1136/jprhc-2013-100863

J Fam Plann Reprod Health Care 2014;40:154. doi:10.1136/jprhc-2014-100881

\section{REFERENCES}

1 Kell P, McMahon K. Comment on 'Abortion care services delivered from a community sexual and reproductive health setting: views of health care professionals'. J Fam Plann Reprod Health Care 2014;40:154.

2 Michie L, Cameron ST, Glasier A. Abortion care services delivered from a community sexual and reproductive health setting: views of health care professionals. J Fam Plann Reprod Health Care 2013;39:270-275. 